\title{
Implementation of Efficient Night Vision Robot on Arduino and FPGA Board
}

\author{
Shivani Sharma \\ Gyancity Research Lab, Gurgaon, India \\ shalu16sharma@gmail.com
}

\begin{abstract}
Night vision robot is robot that can click pictures and make videos during day as well as night. The Project is based on arduino platform. We have used infrared light emitting diodes, light dependent resister, RF module to make remote wireless and a camera. $L D R$ is responsible of clicking pictures and making videos at night. Arduino is an open source microcontroller development board and we have used arduino UNO containing ATmega-328 microcontroller chip. So the idea of our project is to make a prototype of robot with night vision property that can collect the useful information at time of wars. We also implement LDR on FPGA using Verilog Programming Languages and also use output load scaling to make the system energy adequate.
\end{abstract}

Keywords-Night vision robot, ATmega-328 Microcontroller, RF module, Light dependent resistor, IR LEDs.

\section{Introduction}

With the target of the giving fulfillment according to the changing needs of living beings, robotics has been a useful tool that can solve issues. This project focuses on building a RF based spying robot attached with wireless camera that can reduce the human victim. As we were working on Arduino platform, so we decided to make a project that can make use of the Arduino platform in a better way. First of all we decided that we will make a robot whose motion would be controlled manually or externally by using a remote. After this we decided to interface a webcam with our robot that can make video continuously as the robot moves. To enhance its capability we wanted to make it work in the similar way at night also. So, we had to create a module which can help our webcam to make the video in night also. To make it look better we also decided to connect LCD with it showing the motion of robot i.e. left/right/forward/backward. We had all the components needed to fulfill above requirements. So, after this we had a clear picture in our minds what we have to do next. The different values of LDR are taken at different light intensities from serial monitor and the program for Arduino is written accordingly. Analysis of LDR is also done using Xilinx and power readings are noted down at $1 \mathrm{GHz}$ frequency and by varying the value of output load so that we can also work in field of making our project power efficient. Result of LDR simulation on FPGA at frequency $1 \mathrm{GHz}$ for different values of output load is shown in Table 1. FPGA is the best architecture for testing of energy efficient design [9-18]. 


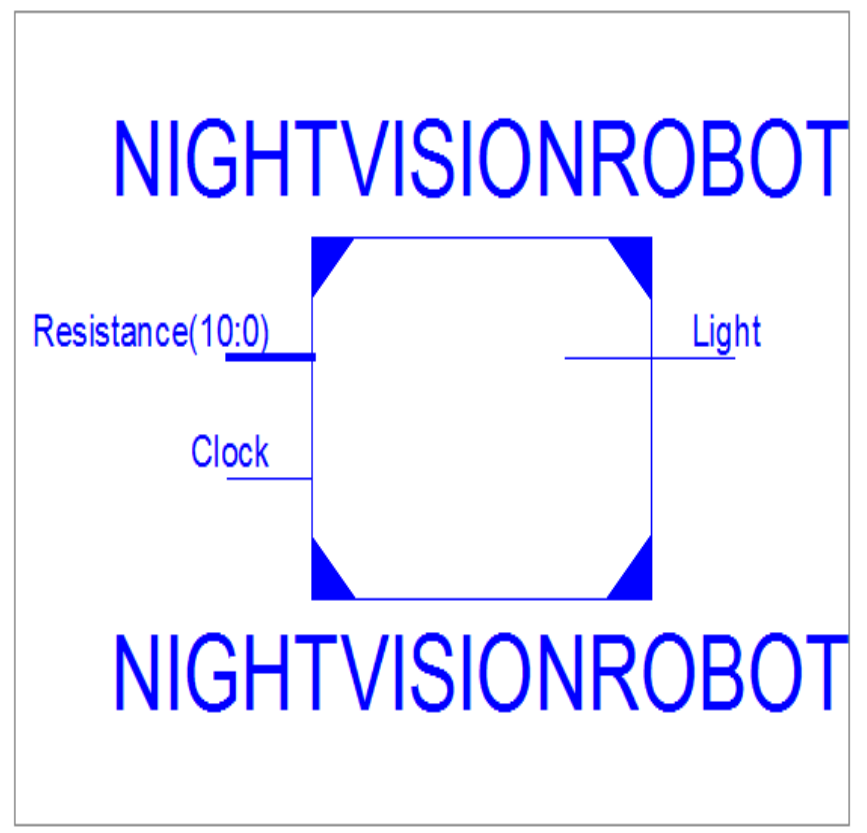

Figure 1: Schematic of LDR using Xilinx on FPGA

There are many energy efficient techniques used in low power electronics design. These are CTHS [9], IO standards [10], Mobile DDR IO standard [11], Voltage Scaling [12], Frequency Scaling [13], CGVS [14], Mapping [15], Thermal Mechanics [16], and Ambient Temperature Scaling [18]. In order to make energy efficient night vision robot, we simulate and implement LDR on FPGA as shown in Figure 1 suign output load scaling.

Table 1:Power reading of LDR for different values of output load

\begin{tabular}{|c|c|c|c|c|c|}
\hline Output Load $(\mathrm{pF})$ & 5 & 100 & 200 & 2000 & 5000 \\
\hline Clocks & 0.014 & 0.014 & 0.014 & 0.014 & 0.014 \\
\hline Logic & 0.000 & 0.000 & 0.000 & 0.000 & 0.000 \\
\hline Signals & 0.000 & 0.000 & 0.000 & 0.000 & 0.000 \\
\hline IOs & 0.007 & 0.008 & 0.010 & 0.036 & 0.081 \\
\hline Leakage & 0.707 & 0.707 & 0.708 & 0.708 & 0.709 \\
\hline Total Power & 0.728 & 0.730 & 0.731 & 0.759 & 0.804 \\
\hline
\end{tabular}

There is no change is clock power, logic power, signal power with change in output load capacitance. There is significant change in IOs power and minor change in leakage power with capacitance scaling. The result in Table 1 concludes that we can save power of LDR if we control output load.

\section{Related Work}

Related work refers to the similar review or research papers that have been published in the past on a related topic as ours. The parameter by which it is judged is the similarity in the concept of the paper and the components used to realize it. In this particular section, we strive to differentiate our paper from the other such already existing ones by giving valid differences and similarities.

\section{A. Road detection at night based on planar reflection model[1]}

This particular paper finds it application during night and is used for the detection of road through pixels only during night. It is very similar to our project as our project also 
highlights the same concept. They are both surveillance robots which detect at night. But the road detection project works on an entirely different principle, that is, a planar reflection model and use a pixel based clarification after which the algorithms are compared.

\section{B. Front \& rear vehicle tracking and observation in the day and night using vision \& sound navigation and ranging sensor fusion [2]}

The above mentioned paper is about a vehicle detection project which detects vehicles in front and the back using image sensors and sonar sensors over a distance of ten meters. It is similar to our project since both night vision robot, and vehicle detection robot are used for seeing things at night but the sensors [17] used by us are light dependent resistor while they have used sonar sensors which are based on the sound reflection theory.

\section{Mono-camera robotic system for tracking moving objects[3]}

The above mentioned project has used arduino as its platform and involves camera interfacing just like our project. They have also used MATLAB for image processing whereas we have interfaced our camera and also placed sensors such as light dependant resistors which activate the infrared LED's to enable the camera to detect objects at night. This is how our project is different from the above mentioned.

\section{Human action recognition for night vision using temporal templates with infrared camera [4]}

The above project is used to recognize human movements at night using thermal infrared camera. While our project uses infrared light emitting diodes which are comparatively much cheaper. Another difference is that our robot is mobile and can detect any sort of movement, not only the human movement during night. The cost effectiveness is a major advantage over the above mentioned paper.

\section{E. Night vision pedestrian detection using a forward-looking Infrared camera} [5]

This paper is on a device that is used for detecting the moving pedestrians during night time while night vision robot detects any movement that takes place in the vicinity of the robot. We have used infrared light emitting diodes and a simple camera while they have made use of an infrared camera.

\section{Components Used}

\section{A. Arduino Board}

We have used Arduino Uno for our project of night vision robot. It has Atmega 328 microcontroller and has 14 pins for digital input and output out of which six can be used as PWM output pins. The voltage at which it operates is $5 \mathrm{~V}$. Flash memory for Arduino is 32 kilobytes and $16 \mathrm{MHz}$ clock speed [6]. We use arduino to interface our LCD (Liquid Crystal Display) and camera.

\section{B. LDR(Light Dependent Resistor)}

It is a resistor whose resistance keeps changing according to the light falling over it, that is, the value of resistance decreases according to the presence of light. It is a bilateral device and conducts in both the directions. It is made up of Cadmium Sulphide. Since the 
project we have made is for night vision, we have used this resistor so that the change in the value of resistance at night enables the infrared light emitting diodes.

\section{a. IR LED}

IR LED stands for infrared light emitting diode. It is also known as IR transmitter and they transmit infrared rays with a wavelength 760 nanometers. It is made up of Aluminum Gallium Arsenide. When glowing, it is not visible to the naked eye but only to the camera. In our project, the camera is surrounded by infrared LED's and as soon as the LDR changes its resistance value, the reflection of IR LED's fall on the camera and the object becomes visible.

\section{b. Wireless Camera}

The camera is used in our project to record the images of the movement during the night time. When the infrared led's glow, the reflection of those led's enable it to see the objects.

\section{C. $L C D$}

LCD stands for liquid crystal display. In our project, we have interfaced it with Arduino. The purpose of LCD is used to display the instructions given to the robot. For exampleif the robot moves left, the display will display left on its screen.

\section{RF module}

We can infer from its name that it works only on radio frequencies. The range for frequencies vary from $30 \mathrm{KHz}-300 \mathrm{GHz}$. The modulation technique used is Amplitude Shift keying. We used the RF module for making our remote which controls the motion of the robot. It helps us in transmitting frequencies over a long distance so it is also used for long distance communication. It has a transmitter and a receiver that operate at 3 $\mathrm{MHz}$ frequency.

\section{E. Actuators:}

Arduino board is connected to two DC motors which are useful in providing forward, reverse, left as well as right motion to the robot.

\section{Working}

The camera that is embedded on the robot can click the pictures in day light as well as in night. For night vision the camera is surrounded by cluster of infrared light emitting diodes. The circuit consists of light dependent resistor. The value of light dependent resistor at night is noted down from serial monitor and is set accordingly in program to make IR LEDs on at night. Light of these IR LEDs through camera makes the object visible in dark and able to click pictures in both daylight and dark.

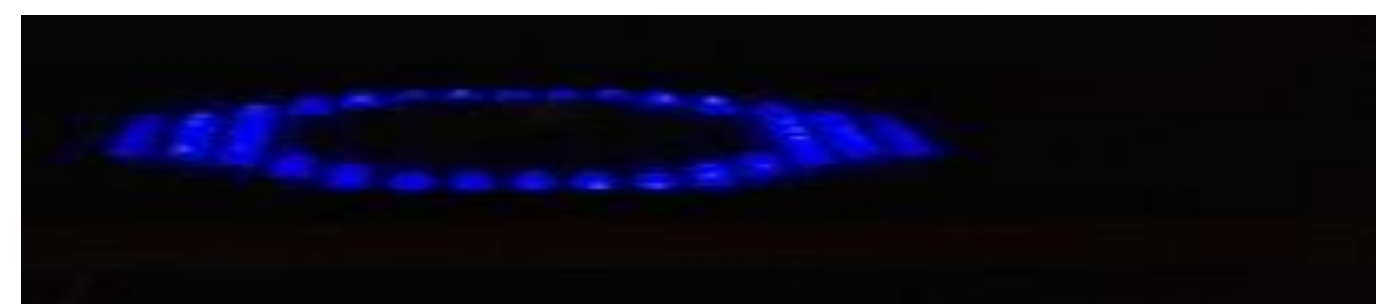

Figure 2: Gleaming of IR LEDs during night using LDR 
Power supply to the robot is provided by arduino and we can also by external source or batteries to make it fast. Motion of the robot is controlled by the program written for arduino in which we have specified all the angles of backward, forward and left right motion including stop angle. This is controlled by a remote that is made wireless with the help of RF module.

\section{Code for Servo Movements and LDR Reading}

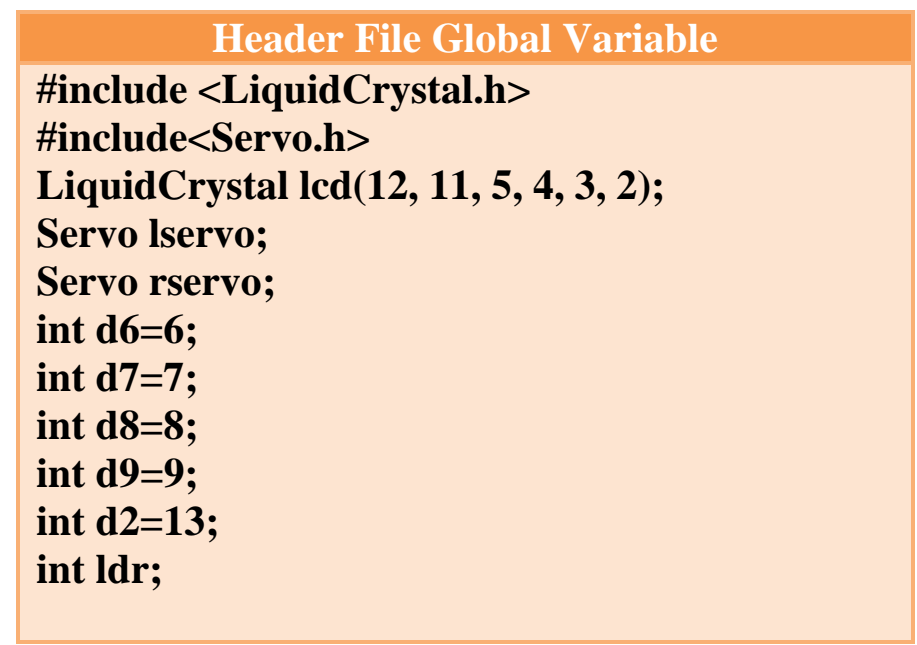

Above mentioned code will define the header files and define the name of servos and also declare the digital pins.

\begin{tabular}{l}
\hline \multicolumn{1}{c}{ SETUP } \\
\hline void setup() \\
\{ \\
Serial.begin(9600); \\
lservo.attach(10); \\
rservo.attach(1); \\
lcd.begin(16,2); \\
pinMode(d6, INPUT); \\
pinMode(d7, INPUT); \\
pinMode(d8, INPUT); \\
pinMode(d9, INPUT); \\
pinMode(ldr,INPUT); \\
pinMode(d2,OUTPUT); \\
\}
\end{tabular}

This code will provide the mode to defined pins whether they are inputs or outputs.

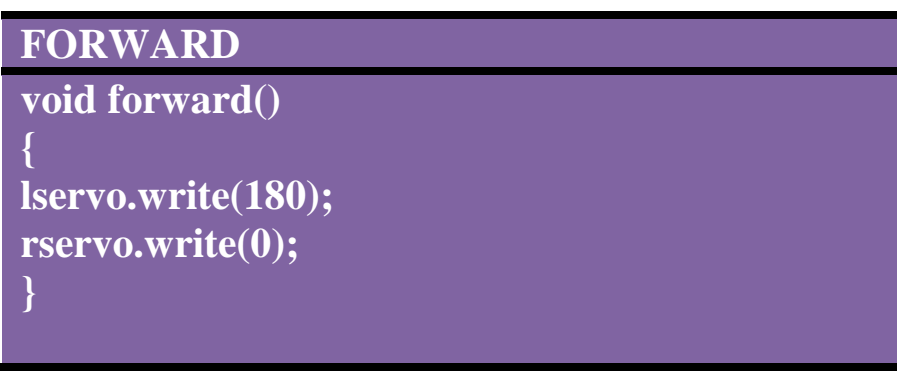

This is for the forward motion of the robot. 
This is for the backward motion of the robot.

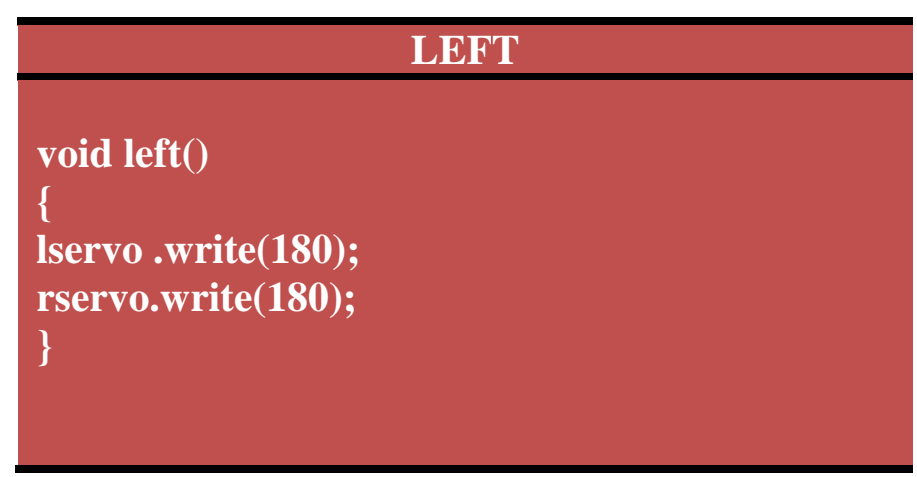

This is for the leftward motion of robot.

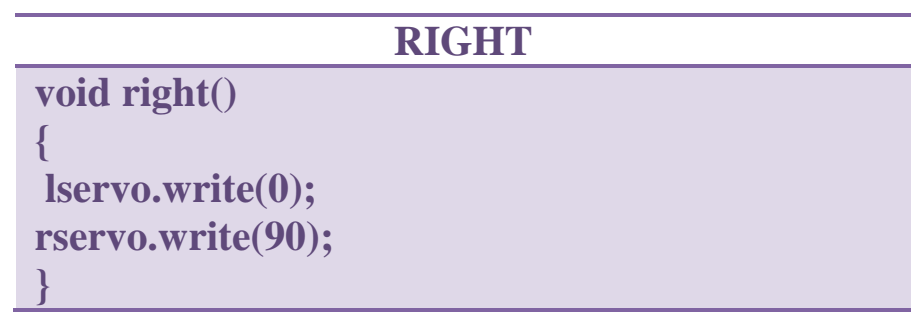

This is for the rightward motion of the robot.

\begin{tabular}{l}
\hline STOP \\
\hline void stopp() \\
\{ \\
lservo.write(90); \\
rservo.write(90); \\
\} \\
\hline
\end{tabular}

This is for halting the robot.

\begin{tabular}{|l|}
\hline \multicolumn{1}{|c|}{ LOOP } \\
\hline void loop() \\
\{ lcd.setCursor(0,0); \\
int d6a=digitalRead(d6); \\
int d7a=digitalRead(d7); \\
int d8a=digitalRead(d8); \\
int d9a=digitalRead(d9); \\
int ldra=analogRead(A0); \\
\hline
\end{tabular}




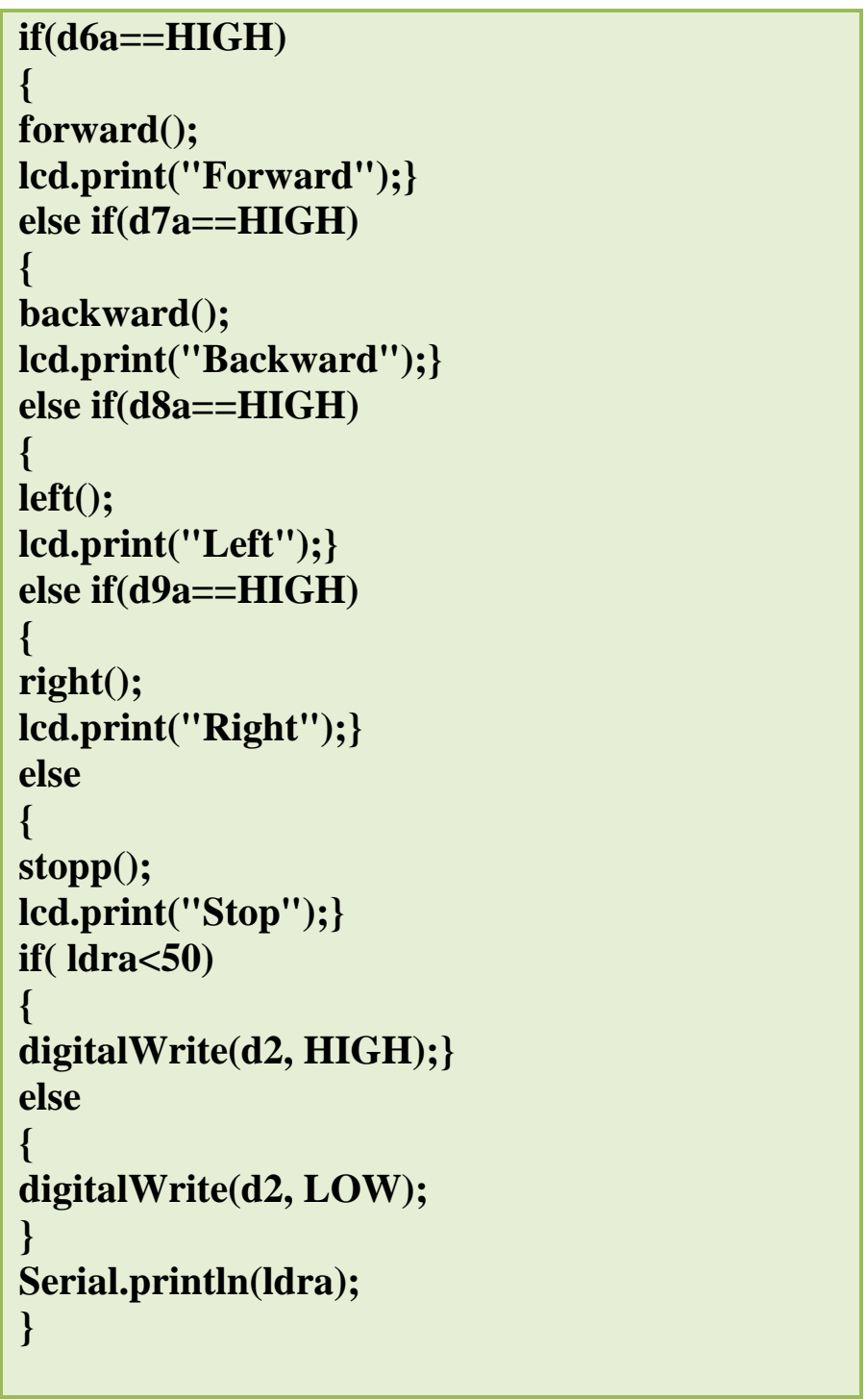

The above mentioned code is for the printing the motion on LCD screen that is the direction of motion of robot.

\section{FUTURE SCOPE}

There are many prototypes made having a robot that can click pictures but the idea of robot that can click pictures or made videos at night using a simple light dependent resistor seems to be quite interesting. This project have a wide scope in future and can have number of enhancements as well that can be done in future. This project is a prototype of night vision robot. This project is designed to establish a robotic vehicle for remote area operations and which is attached to a wireless camera for monitoring purpose. The robot can transmit real time data such as videos or photos in day as well as night also. The wireless robot can be useful for under cover purpose in battle fields. Location of terrorists can suitably detected using such robot. This system can be connected directly to internet by using zigbee with Wi-Fi.[7] By using internet we can control the system via remote location Further the work can be upgraded using Dual Tone Multi Frequency signaling technology. This technique is useful in controlling the robotic device with the help of cell phone and communication range can be improved. These robots can be also used to hit a target with help of the remote by seeing the targets 
on the video. This robot has a great advantage in the field of military. Further the work can be done in field of range it can cover, the duration for which it can make videos and how alert it would be for actions of enemy.GPS system can also be installed to determine the location of the robot. It can be enhanced using Gas sensor which can be used to sensing poisonous gases that leaks in war field [8].

\section{References}

[1] T. Cheng, "Road detection at night based on a planar reflection model." Information and Automation, International Conference on. IEEE, 2013.

[2] K.S.Yong. "Front and rear vehicle observation and tracking in the day and night times using vision and sonar sensor fusion." International Conference on Intelligent Robots and Systems, IEEE, 2005.

[3] D. Gornea, "Mono-camera robotic system for tracking moving objects." 9th Conference on Industrial Electronics and Applications IEEE, 2014

[4] H. Eum, "Human action recognition for night vision using temporal templates with infrared thermal camera." URAI. 2013.

[5] H. Sun, C. Wang, and B. Wang. "Night vision pedestrian detection using a forward-looking infrared camera." International Workshop on Multi-Platform/Multi-Sensor Remote Sensing and Mapping. IEEE, 2011.

[6] Arduino description available at: https://www.arduino.cc/en/Main/ArduinoBoardUno

[7] L. Mehta, P. Sharma. "Spy Robot with Moving Wireless Video Camera \& Ultrasonic Sensor", International Journal of Research in Engineering Technology and Management, June 2014.

[8] G. U. Meshram, and S. Borkar. "Design and Implementation of Robot Motion with IR Wireless Camera.", International Journal on Recent and Innovation Trends in Computing and Communication, pp. 028-031

[9] T. Kumar, B. Pandey, S. H. A. Musavi, N. Zaman,“CTHS Based Energy Efficient Thermal Aware Image ALU Design on FPGA", Springer Wireless Personal Communications, An International Journal, ISSN, Vol.83, No.1, July 2015.

[10] S. H. A. Musavi, B. S. Chowdhry, T. Kumar, B. Pandey, W. Kumar, "IoTs Enable Active Contour Modeling Based Energy Efficient and Thermal Aware Object Tracking on FPGA", Springer Wireless Personal Communications, Vol.82, No.3, pp.1-15, 20 May 2015.

[11] T. Kumar, B. Pandey, T. Das, and B. S. Chowdhry, "Mobile DDR IO Standard Based High Performance Energy Efficient Portable ALU Design on FPGA", Springer Wireless Personal Communications, An International Journal, Volume 76, Issue 3 (2014), Page 569-578.

[12] S. M. M. Islam, B. Pandey, S. Jaiswal, M. M. Noor and S. M. T. Siddiquee, "Simulation of Voltage Scaling Aware Mobile Battery Charge Controller Sensor on FPGA", "Advanced Materials Research" pp 798-802, February 2014.

[13] R. Kaur, S. M. M. Islam, T. Kumar, B. Pandey, M. M. Noor and S. M. T. Siddiquee, "Frequency Scaling Based Green Mobile Battery Charge Controller Sensor Design on FPGA", "Advanced Materials Research, pp. 1057-1062.

[14] T. Das, T. Kumar, S. M. M. Islam, B. Pandey, M A. Rahman and M. M. Noor, "Low Power Devnagari Unicode Checker Design Using CGVS Approach", "Advanced Materials Research", Vol. 984 - 985, pp. 1282-1285.

[15] N. Singh, T. Kumar, T. Das, B. Pandey "Mapping Based Power Optimized Counter Design on Field Programmable Gate Array", "Advanced Materials Research", Volumes 984 - 985, pp. 1085-1088.

[16] B. Pandey, T. Kumar, T. Das and J. Kumar, "Thermal Mechanics Based Energy Efficient FIR Filter for DSP", Applied Mechanics and Materials Journal,, pp. 65-70, August 2014.

[17] B. S. Chowdhry, B. Pandey, T. Kumar, T. Das, S. Thakur, "Frequency, Voltage and Temperature Sensor Design for Fire Detection in Very Large Scale Integration Circuit on Field Programmable Gate Array", Springer Communications in Computer and Information Science,Sep. 2014.

[18] R. Saini, N. Bansal, M. Bansal, L. Kalra, B. Pandey and D. M. A. Hussain, "Ambient Temperature Based Thermal Aware Energy Efficient ROM Design on Field Progammable Gate Array", Advanced Materials Research, Trans Tech Publications, pp.467-470 